\title{
Simulation of the Freeman Model of the Olfactory Cortex: A Quantitative Performance Analysis for the DSP Approach
}

\author{
Mustafa C. Ozturk, Jose C. Principe, Bryan Davis, Deniz Erdogmus \\ Electrical Engineering Department, University of Florida, Gainesville, FL 32611, USA
}

\begin{abstract}
It has been shown that a framework composed of digital signal processing (DSP) elements can be used to simulate and study Freeman's model of the biologically realistic olfactory cortex. In this paper, based on impulse invariant transformation, a DSP environment has been developed corresponding to the original continuous-time dynamical system. The performance of the DSP system is quantitatively evaluated by comparing with the original system, which is implemented using the traditional Runge-Kutta integration technique. The discrete-time architecture is shown to have high performance in approximating the dynamical behavior of the original system.
\end{abstract}

\section{INTRODUCTION}

Researchers have long been interested in understanding how the brain works. Freeman [1], who developed a biologically realistic dynamical model for the olfactory cortex that conforms to the anatomical and physiological properties of the olfactory system, made a major contribution to the understanding of the behavior of biological neural networks. The mentioned model is a complex dynamical system built on the principle of coupled nonlinear oscillators, where the core of the architecture is a simple second order nonlinear dynamical element. According to Katchalsky these basic units are organized in the model in a hierarchy of levels designated by K0, KI, KII, and KIII [1]. The lowest level in the hierarchy of complexity, the $\mathrm{K} 0$ set, is identified by the second order dynamics given by

$$
\frac{1}{a b} \ddot{y}(t)+\frac{a+b}{a b} \dot{y}(t)+y(t)=u(t)
$$

where $a$ and $b$ are real time constants determined experimentally, $u(t)$ is the forcing input, and $y(t)$ is the filter output [1][2].

A continuous time differential equation such as (1) has to be discretized in order to simulate in a digital computer environment. Previously, a digital system corresponding to Freeman's original dynamical system was developed based on the impulse invariant transformation [3]. Gamma filter architecture [4] was employed to form the basis of this approach to approximately realize the olfactory system in a digital setup.

In this paper, the performance of the gamma filter architecture is evaluated quantitatively through an output comparison with the original system, which is realized

This work was partially supported by ONR Grant N00014-01-0405. using Runge-Kutta integration [6]. In addition, an analysis of the performance of the direct implementation of the discretized system transfer function is carried out. The latter approach, which uses impulse invariance, reduces computational load, since the system identification stages necessary for designing the gamma filter approximation are avoided. It turns out that, in fact, the direct implementation results in better approximation of the continuous time olfactory system at the KII level according to the performance measure that is assumed.

\section{OVERVIEW OF THE FREEMAN MODEL}

Freeman proposed a biologically plausible model for the olfactory cortex, which is built from basic second order nonlinear elements in a hierarchy of levels designated by $\mathrm{K} 0$, KI, KII, and KIII [1]. The basic building block of the model is the $\mathrm{K} 0$ set, which is depicted in Fig. 1. This set has no functional interconnections among the elements of neural assembly. Every K0 unit can accept several inputs that are weighted and summed, and then convolved with a linear time invariant system defined by the second order dynamics $H(s)$. The output of the linear dynamics is then shaped by the nonlinear function, which is experimentally determined to be

$$
Q(x)=\left\{\begin{array}{cl}
q\left(1-\exp \left(-\left(e^{x}-1\right) / q\right)\right) & \text { if } x \geq x_{0} \\
-1 & \text { if } x<x_{0}
\end{array}\right.
$$

The second level in the hierarchy, the KI set, is built from K0 units that interact through lateral inhibitory (-) or excitatory $(+)$ feedback. An example $\mathrm{KI}$ element is demonstrated in Fig. 2, where the circles denote a K0 unit. A KI network can be formed by fully interconnecting KI sets. The inhibitory/excitatory connection strengths are determined by the weights of the network.

The third level in the hierarchy, the KII set, is the most interesting and important building block of the olfactory system, since it is an oscillator controlled by the input. The response of the KII set to an impulse input is a damped oscillation whereas with a sustained input, the output oscillation is maintained as long as the input remains. The KII set is built from two KI sets, interacting through both excitatory and inhibitory connections. This architecture is shown in Fig. 3, where the circles again denote K0 sets. A KII network can be formed by fully interconnecting a number of KII sets.

The final Katchalsky level is the KIII network and represents the olfactory cortex. The KIII model has several layers formed by $\mathrm{K} 0, \mathrm{KI}$, KII sets that resemble the main 
parts of the central olfactory system: the bulb (OB), anterior nucleus (AON), and prepyriform cortex (PC). For the full model of the KIII network, we refer the reader to [2].

For the purposes of this paper, the focus of numerical simulations will be on the reduced KII set, which behaves similar to the full KII set shown in Fig. 3. The reduced KII set is formed by connecting two K0 sets, one excitatory and one inhibitory. If the coupling weights are selected properly, the reduced KII is an oscillator controlled by the input [1].

The reduced KII architecture is demonstrated in Fig. 4, where the two K0 units are denoted by M (for mitral cell) and $\mathrm{G}$ (for granular cell). In this model, the mitral cell takes the external input $I(t)$ and the coupling strengths between $\mathrm{M}$ and G are controlled by the two weights $K_{m g}>0$ (excitatory) and $K_{g m}<0$ (inhibitory). The external input is allowed to be an impulse, a step, or arbitrarily time-varying. The governing equations for the reduced KII set are

$$
\begin{aligned}
& \frac{1}{a b} \ddot{y}_{m}(t)+\frac{a+b}{a b} \dot{y}_{m}(t)+y_{m}(t)=K_{g m} Q\left(y_{g}(t)\right)+I(t) \\
& \frac{1}{a b} \ddot{y}_{g}(t)+\frac{a+b}{a b} \dot{y}_{g}(t)+y_{g}(t)=K_{m g} Q\left(y_{m}(t)\right)
\end{aligned}
$$

where $y_{m}$ and $y_{g}$ denote the output of the linear dynamic portions of the $\mathrm{K} 0$ units for $\mathrm{M}$ and $\mathrm{G}$, respectively [2].

\section{DigitAl IMPLEMENTATION APPROACHES}

In order to simulate continuous time equations in computer environment, discretization is employed. The traditional Runge-Kutta integration techniques [6] are not suitable for real-time implementations of such neural networks. However, in the design stage, the Runge-Kutta solution of the Freeman model can be utilized as a benchmark to determine performance.

\section{A. System Identification with Adaptive Gamma Filter}

Previously, DSP and system identification techniques based on the gamma filter approximation architecture were used to achieve discretized approximations to the olfactory system [3]. The gamma filter implementation requires the decomposition of the desired impulse response using gamma basis functions. This filter structure is based on the first-order generalized unit delay whose dynamics are defined as [4]

$$
G(z)=\frac{\mu}{z-(1-\mu)}
$$

A second-order gamma filter structure is shown in Fig. 5. It was determined that the MSE-optimal weights for this second-order gamma filter to best approximate the continuous-time system impulse response are $w_{0}=-2.9 \times 10^{-3}$, $w_{1}=1.245 \times 10^{-1}, w_{2}=0.9016$. The feedback parameter, which determines the average memory depth of the filter, was found as $\mu=0.237 \times 10^{-1}$ [3].

Since gamma filter is an approximation to the desired transfer function, it does not preserve the impulse response exactly. Consequently, the step response is not also

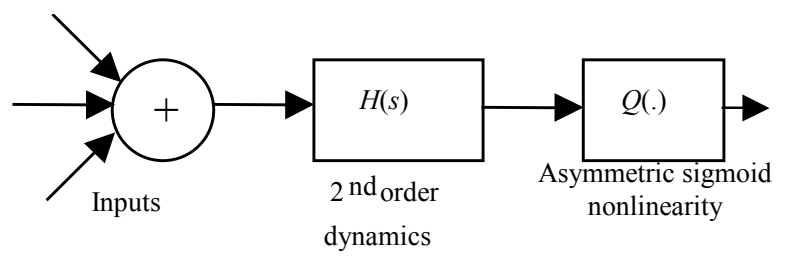

Figure 1. The K0 set.
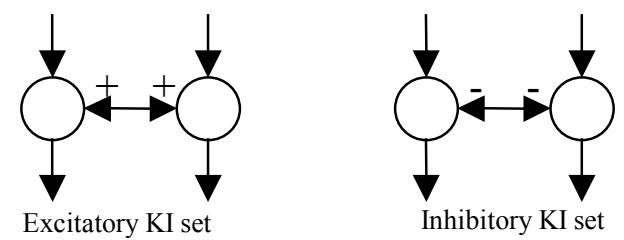

Figure 2. The KI set.

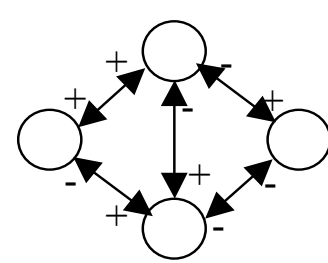

Figure 3. The KII set.

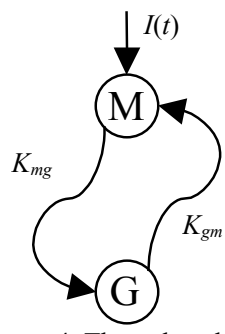

Figure 4. The reduced KII set. approximated accurately. The step response accuracy is particularly important in the discretization of the Freeman model, because the inputs to the system are usually on-off type square signals. There are at least two reasons for the significance of this type of input patterns: biological stimuli may be represented as existent or non-existent, and piecewise constant inputs lend more tractable dynamics that are easier to analyze and understand. In addition, since the system exhibits a tightly coupled oscillatory behavior, there is a high probability that any error will be amplified in time.

\section{B. Direct Discretization with Impulse Invariance}

Alternatively, it is possible to design a discrete-time approximation to the olfactory system using the direct discretization approach. This approach eliminates the need to perform system identification and adaptive filter optimization tasks, reducing design complexity. An improvement in the performance of the approximation of the original continuoustime system by its discrete-time equivalent is expected, since the exact transfer function is used to generate the discretized approximation instead of learning the approximation with a finite-tap adaptive gamma filter ${ }^{1}$.

Consider the linear dynamical part of the $\mathrm{K} 0$ set defined by (1). The input-output transfer function is simply

$$
H(s)=\frac{a b}{(s+a)(s+b)}=a b\left(\frac{s+a}{b-a}+\frac{s+b}{a-b}\right)
$$

\footnotetext{
${ }^{1}$ Although the gamma filter is IIR, it is considered a finite-tap filter because it contains only a finite number of generalized delay operators.
} 
which leads to a time-domain signal of

$$
h(t)=a b\left(\frac{e^{-a t}}{b-a}+\frac{e^{-b t}}{a-b}\right)
$$

Using the impulse invariant transformation technique [5] with sampling period $T_{s}$, we obtain that the discrete approximation filter should possess the following impulse response.

$$
h[n]=T_{S} h\left(n T_{S}\right)=T_{S} a b\left(\frac{e^{-a n T_{s}}}{b-a}+\frac{e^{-b n T_{s}}}{a-b}\right)
$$

Letting $\alpha_{1}=e^{-a T_{s}}, \alpha_{2}=e^{-b T_{s}}, c_{1}=T_{s} a b /(b-a)$, and $c_{2}=T_{S} a b /(a-b)$, the transfer function of the equivalent digital system is finally determined to be

$$
H(z)=\frac{c_{1}}{1-\alpha_{1} z^{-1}}+\frac{c_{2}}{1-\alpha_{2} z^{-1}}
$$

In general, discretization of continuous-time signals is susceptible to aliasing. However, in this case, the continuoustime system exhibits a low-pass filter behavior, since the poles $a$ and $b$ are positive real numbers. Therefore, aliasing error can be controlled by manipulating $T_{s}$ [5].

Implementation of the transfer function in (7) can be achieved using, for example, the parallel realization approach, which is shown in Fig. 6. This architecture is supported by many standard commercial DSP software or hardware products and can be efficiently implemented to operate accurately in real-time.

\section{Comparison of Impulse Responses}

In this section, we present a comparison of the impulse and step responses of three systems: continuous-time original, discrete-time gamma filter approximation and discrete-time impulse-invariant approximation. The impulse responses are shown in Fig. 7 and the step responses are in Fig. 8. As a quantitative measure of similarity, we employ the meansquare-error (MSE) between the impulse responses of the discrete-time approximations and the original filter.

$$
M S E=\sum_{n}\left(h_{d}[n]-h_{c}\left(n T_{S}\right)\right)^{2}
$$

where $h_{d}$ and $h_{c}$ denote the impulse responses of the discrete and continuous-time filters. The impulse response MSE for the gamma filter and the impulse invariant filter approximations are found as $4.73 \times 10^{-5}$ and $4.72 \times 10^{-6}$, respectively. The noticeable one order of magnitude difference is mainly due to the approximation errors incurred on the gamma filter due to the finite tap length.

\section{Quantitative Performance Analysis}

The overall Freeman is a highly complex nonlinear dynamical system with coupled oscillations. Hence, the discrepancy between the dynamics of the linear portion of the basic $\mathrm{K} 0$ sets alone is not an accurate descriptor of the global

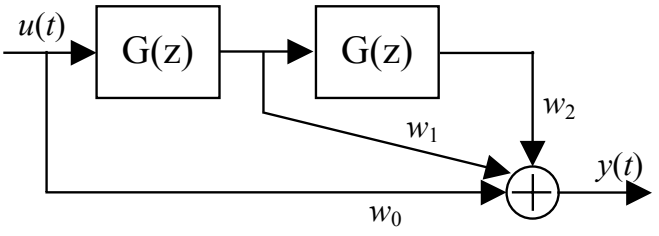

Figure 5. Gamma filter implementation of LTI dynamics.

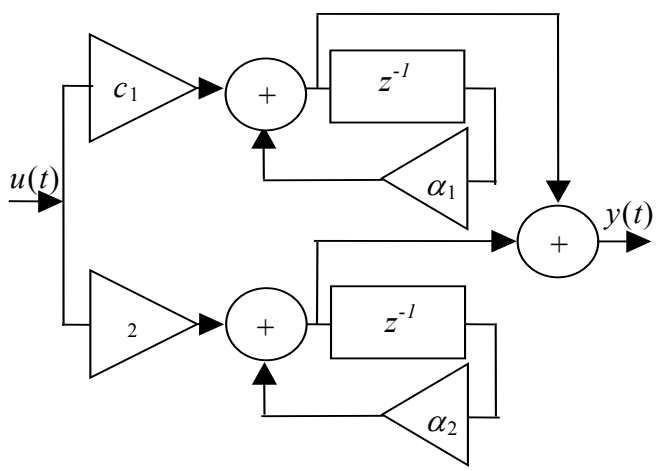

Figure 6. Parallel implementation of LTI dynamics.

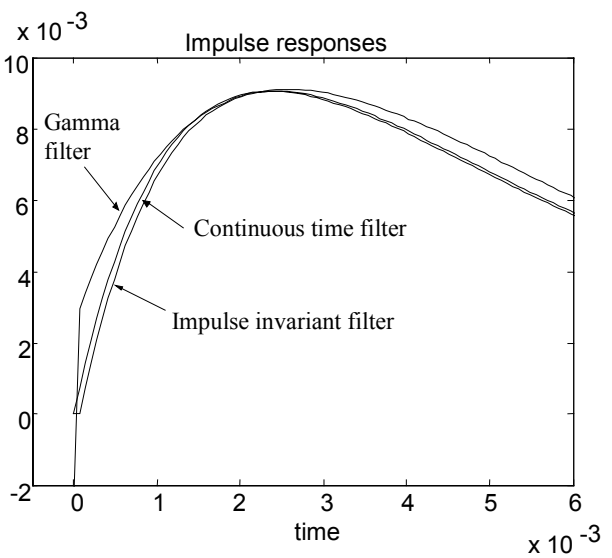

Figure 7. Impulse responses of the three filters.

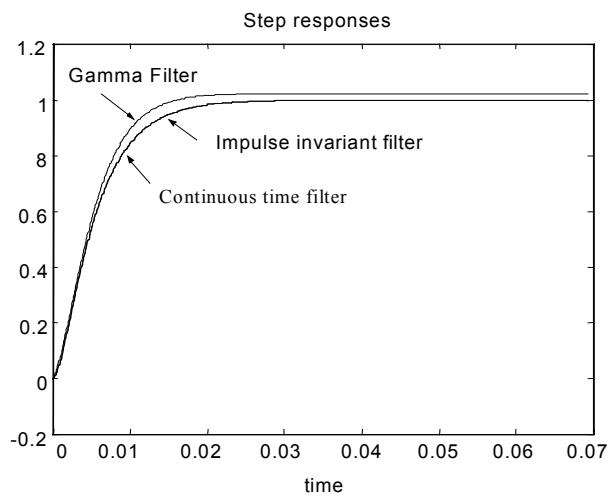

Figure 8. Step responses of the three filters.

behavior. In order to investigate the effect of the discrete-time approximation errors on the nonlinear behavior, we investigate the performance of the approximations on the reduced KII network, which might be considered as the smallest nonlinear unit representative of the global KIII dynamics. 
In particular, the reduced KII set, for specific choices of the connection weights, possesses a fixed point and a limit cycle. The operation mode of the coupled system is controlled by the on-off input signal. Moreover, we can achieve a comprehensive and decisive performance analysis, since the reduced KII set has only two parameters to be controlled: $K_{m g}$ and $K_{g m}$. In the following numerical examples, the output of the reduced KII set is defined to be the output of the mitral K0 set (see Fig. 4).

For comparison, the continuous-time system is simulated using the ODE45 built-in Runge-Kutta integration technique in Matlab $^{\circledR}$ [6]. The digital network simulations are performed using the commercial software package Neurosolutions ${ }^{\circledR}$ [7], which is specialized for neural network simulations. Neurosolutions ${ }^{\circledR}$ has all the basic building blocks necessary to implement the discretized Freeman model. The software has a graphical user-interface, which allows building an icon-based reduced KII model.

Ideally, with the input on, if the connection weights are appropriate, the KII model will exhibit sustained oscillations. Therefore, the digital simulators are expected to behave exactly the same way as the original system. The performance of the digital systems are measured by the distance between the points in the parameter space $\left[K_{m g}, K_{g m}\right]$ at which bifurcation occurs. The following measure is defined to provide a percentage-parameter-error (PPE) to quantify the approximation quality.

$$
P P E=\frac{K_{c}-K_{d}}{K_{c}} \times 100 \%
$$

In (9), $K_{c}$ is the $\left|K_{g m}\right|$ (or $\left|K_{m g}\right|$ ) value at which the continuoustime system switches from non-oscillatory to oscillatory behavior while $K_{m g}$ (or $K_{g m}$ ) is kept fixed. $K_{d}$ is, similarly, the parameter value for the discrete-time approximation under consideration (either the gamma filter or the impulse invariant filter).

In the following simulations, the input is assumed to be on continuously and the sampling frequency for the impulse invariant filter is taken as 20 times the faster pole in the continuous-time linear dynamics for alias-free approximation [5]. Fig. 9, 10, 11 show the outputs of the continuous-time and discrete-time reduced KII sets where $K_{m g}$ is kept constant and $K_{g m}$ is varied until bifurcation is observed. According to the PPE measure, the approximation errors of the gamma and impulse invariant filter approximations are determined as

$$
\begin{aligned}
& \text { PPE }(\text { gamma })=\frac{4.27-3.22}{4.27} \times 100 \%=24 \% \\
& \text { PPE }(\text { imp.inv })=\frac{4.27-3.95}{4.27} \times 100 \%=7.49 \%
\end{aligned}
$$

Clearly, the PPE for both approaches will decrease as the sampling frequency of the impulse response is increased. For each different sampling frequency the gamma filter method requires solving a linear system identification problem with a nonlinear optimization index. The impulse invariant filter method, however, is easily modified for the new sampling
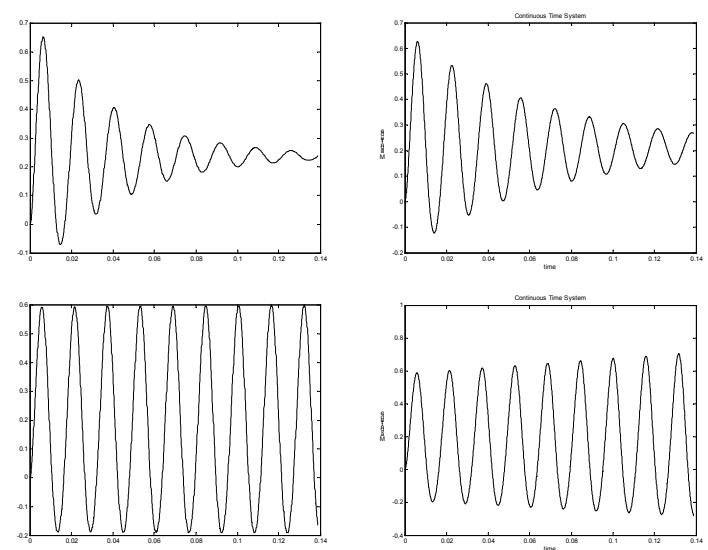

Figure 9. Behavior of continuous-time reduced KII set for $K_{m g}=1$ switches from a point attractor to a limit cycle as $K_{g m}=-3,-3.5,-4.25,4.5$. Bifurcation occurs at $K_{g m}=-4.27$.
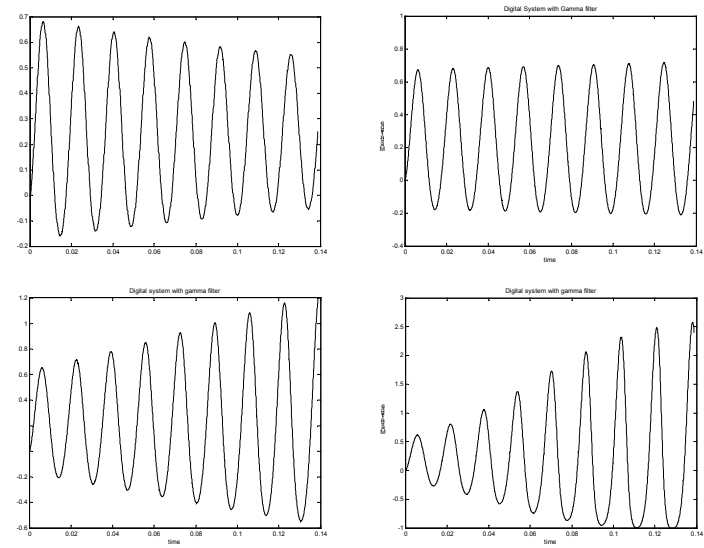

Figure 10. Behavior of gamma filter approximation of reduced KII set for $K_{m g}=1$ switches from a point attractor to a limit cycle as $K_{g m}=-3,-3.25,-3.5$, 4.25. Bifurcation occurs at $K_{g m}=-3.22$.
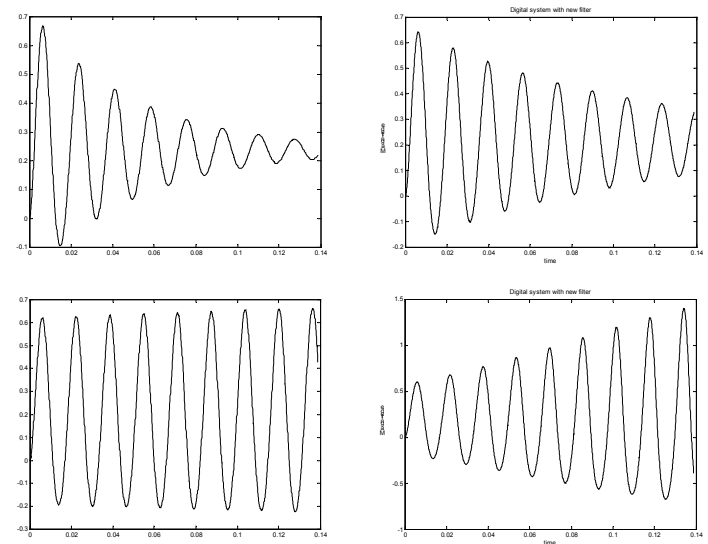

Figure 11. Behavior of impulse invariant filter approximation of reduced KII set for $K_{m g}=1$ switches from a point attractor to a limit cycle as $K_{g m}=-3,-3.5$, $-4,-4.5$. Bifurcation occurs at $K_{g m}=-3.95$.

frequency by simply reevaluating the values for the discrete transfer function parameters in (7). Therefore, only the performance of the impulse invariant method is investigated versus increasing sampling frequency. The performance 
(PPE) of the impulse invariant method versus $1 / T_{s}$ is shown in Fig. 12. On the other hand, increasing sampling frequency will increase the computation requirements of the discretized systems. This creates a trade-off between modeling accuracy and computation speed controlled by the sampling period.

In general, the dynamic behavior of the reduced KII sets for the original continuous-time system, the gamma filter approximation, and the impulse invariant approximation are segmented into two regions in the parameter space by the boundaries shown in Fig. 13. As observed from this plot, the bifurcation boundary for the gamma filter approximation is farther to the bifurcation boundary of the original system than the boundary of the impulse invariant approximation. This analysis assures the better performance of the impulse invariant approximation compared to the gamma filter approach.

\section{CONCLUSIONS}

Freeman's dynamical model for the olfactory cortex provides an insight to how the brain operates in processing information from external stimuli. The general trend in neural engineering is to incorporate more biologically realistic neural network models to the solution of engineering problems of various natures. In this respect, this dynamical model of the olfactory cortex, an area responsible for processing sensory information, offers a potential improvement in designing innovative information processing systems. A first step to this end is designing efficient, accurate, and fast (real-time) approximations of this continuous-time, nonlinear dynamical system.

In this paper, this problem is tackled in a DSP framework, where two discrete-time models that approximate the behavior of the actual dynamics are investigated. A quantitative performance comparison of the two approximation methods, namely the gamma filter and the impulse invariant filter approaches, is provided.

Experiments performed on the relatively simple reduced KII set demonstrated the better accuracy of the impulse invariant filter design approach. Future work will expand the results provided herein to the more complicated KIII network. A more difficult problem that is yet to be solved is the design of a read-out protocol that will allow the use of this system as a dynamical associative memory.

\section{REFERENCES}

[1] Y. Yao, W.J. Freeman, B. Burk, "Central Pattern Generating and Recognizing in Olfactory Bulb: A Correlation Learning Rule," Neural Networks, vol. 1, pp. 277-288, 1988.

[2] Y. Yao, W.J. Freeman, "Model of Biological Pattern Recognition and Spatially Chaotic Dynamics," Neural Networks, vol.3, pp. 153-170,1990.

[3] V.M. Tavares, Design and Implementation of a Biologically Realistic Olfactory Cortex in Analog VLSI,

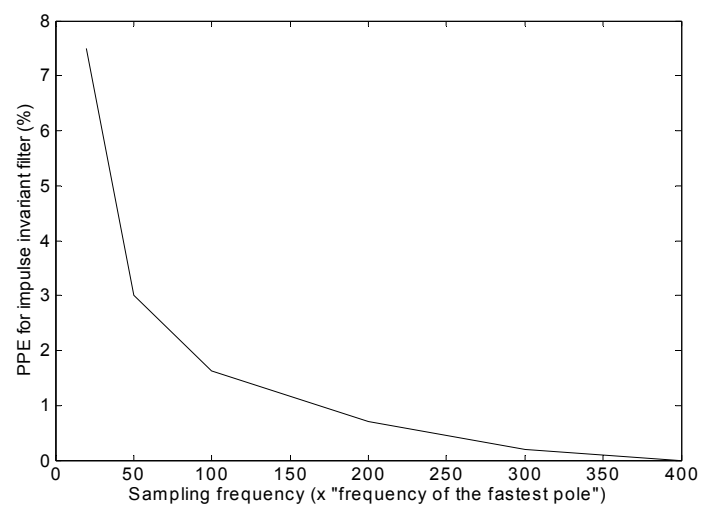

Figure 12. PPE of impulse invariant method versus sampling frequency.

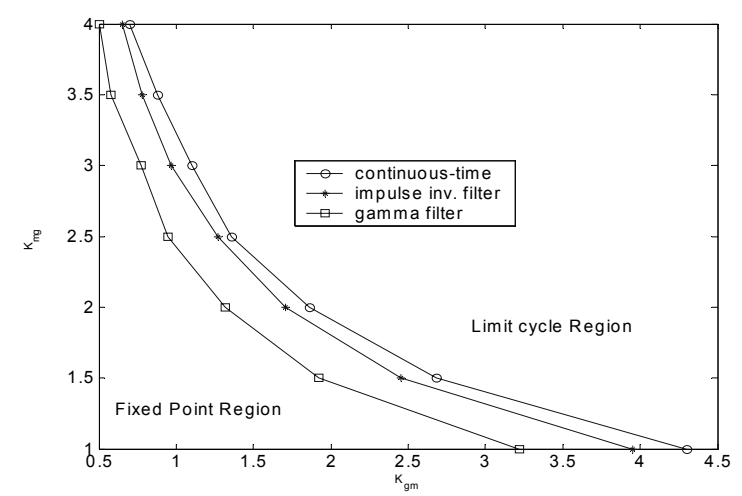

Figure 13. Boundaries between oscillatory and convergent dynamics in the parameter space of the reduced KII set.

Ph.D. Dissertation, University of Florida, Gainesville, FL, 2001.

[4] B. De Vries, Temporal Processing with Neural Networks-The Development of the Gamma Model, Ph.D. Dissertation, University of Florida, Gainesville, FL, 1991.

[5] A.V. Oppenheim, R.W. Schafer, Discrete Time Signal Processing, Prentice Hall, Englewood Cliffs, NJ, 1989.

[6] The MathWorks, Inc. 'http://www.mathworks.com"

[7] NeuroDimension, Inc. 'http://www.nd.com” 